\title{
Feeding spinach thylakoids to rats modulates the gut microbiota, decreases food intake and affects the insulin response
}

\author{
Caroline Montelius ${ }^{1} \dagger$, Nadia Osman $^{1} \dagger$, Björn Weström ${ }^{2}$, Siv Ahrné ${ }^{3}$, Göran Molin ${ }^{3}$, Per- Åke Albertsson ${ }^{4}$ \\ and Charlotte Erlanson-Albertsson ${ }^{1}$ * \\ ${ }^{1}$ Department of Experimental Medical Science, Lund University, Lund, Sweden \\ ${ }^{2}$ Department of Biology, Lund University, Lund, Sweden \\ ${ }^{3}$ Department of Food Technology, Engineering and Nutrition, Lund University, Lund, Sweden \\ ${ }^{4}$ Department of Biochemistry and Structural Biology, Lund University, Lund, Sweden
}

(Received 19 April 2012 - Final revision received 2 November 2012 - Accepted 6 November 2012)

Journal of Nutritional Science (2013), vol. 2, e20, page 1 of 9

doi:10.1017/jns.2012.29

Abstract

Thylakoid membranes derived from green leaf chloroplasts affect appetite-regulating hormones, suppress food intake, reduce blood lipids and lead to a decreased body weight in animals and human subjects. Thylakoids also decrease the intestinal in vitro uptake of methyl-glucose in the rat. The aim of this study was to investigate the effect of dietary thylakoids on the gut microbiota composition, mainly the taxa of lactobacilli and bifidobacteria, in rats fed either a thylakoid-enriched diet or a control diet for $10 \mathrm{~d}$. At the same time, a glucose-tolerance test in the same rats was also performed. Food intake was significantly decreased in the thylakoid-fed rats compared with the control-fed rats over the 10-d study. An oral glucose tolerance test after $10 \mathrm{~d}$ of thylakoid- or control-food intake resulted in significantly reduced plasma insulin levels in the thylakoid-fed rats compared with the control-fed rats, while no difference was observed for blood glucose levels. Analysis of gut bacteria showed a significant increase of lactobacilli on the ileal mucosa, specifically Lactobacillus reuteri, in the rats fed the thylakoid diet compared with rats fed the control diet, while faecal lactobacilli decreased. No difference in bifidobacteria between the thylakoid and control groups was found. Analyses with terminal restriction fragment length polymorphism and principal component analysis of faeces demonstrated different microbial populations in the thylakoid- and control-fed animals. These findings indicate that thylakoids modulate the gut microbial composition, which might be important for the regulation of body weight and energy metabolism.

Key words: Colon: Small intestine: Obesity: Lactobacilli: Quantitative PCR

Today, over one billion adults are overweight with BMI between 25 and $30 \mathrm{~kg} / \mathrm{m}^{2}$, and more than 300 million are obese, with BMI $>30 \mathrm{~kg} / \mathrm{m}^{2}$, worldwide $^{(1)}$. Overweight and obesity are strongly associated with type 2 diabetes, hyperlipidaemia, atherosclerosis and $\operatorname{CVD}^{(2,3)}$. The increased availability of palatable food, i.e. foods rich in fat and sucrose, in Western society is hypothesised to play a significant role in this global increase of body weight and metabolic disease.

Recent data demonstrate that the gut microbiota may affect lipid metabolism and might therefore be important for the development of obesity and related diseases ${ }^{(4,5)}$. The microbiota can be viewed as a metabolic 'organ' exquisitely attuned to the host's physiology. One function of the microbiota is the ability to process otherwise indigestible components of the diet, such as plant polysaccharides, as well as taking part in nutrient acquisition and energy regulation ${ }^{(6)}$. In recent years, it has been proposed that the composition of the gut microbiota differs between normal-weight, overweight and obese persons ${ }^{(7)}$. Compositional changes of the microbiota have also been demonstrated to be associated with the development of diabetes $^{(8)}$, and for the regulation of fat storage via different gene expressions $^{(9)}$. In recent years, some studies have reported Abbreviations: MW, modified Wilkins-Chalgren; OGTT, oral glucose tolerance test; PCA, principal component analysis; qPCR, quantitative PCR; T-RFLP, terminal restric-
tion fragment length polymorphism.

* Corresponding author: Professor C. Erlanson-Albertsson, fax +46 46 2224022, email charlotte.erlanson-albertsson@med.lu.se

†These authors have contributed equally to the scientific work.

(C) The Author(s) 2013. The online version of this article is published within an Open Access environment subject to the conditions of the Creative Commons Attribution-NonCommercial-ShareAlike licence $<\mathrm{http}: / /$ creativecommons.org/licenses/by-nc-sa/2.5/>. The written permission of 
that lactobacilli and bifidobacteria may be important for bodyweight regulation by acting as an anti-obesity factor ${ }^{(4,5,10,11)}$. Understanding changes in the microbiota, as well as their signalling pathways, provides an opportunity to identify new therapeutic targets for promoting health.

Thylakoids, isolated from the chloroplast membrane of green leaves, contain proteins, lipids and pigments (e.g. chlorophyll and carotenoids). They have been found to increase satiety, decrease hunger signals and promote weight loss in a number of studies, both in human subjects and in animals ${ }^{(12-15)}$. The satiety-promoting effects induced by thylakoids were explained by their interaction with dietary lipids, prolonging the digestion of dietary fat ${ }^{(15)}$. Thylakoids have also been found to create a physical 'barrier' on the mucosal surface of the intestine in vitro, causing a decreased uptake of methyl-glucose and macronutrients over the intestinal wall ${ }^{(16)}$.

Since thylakoids interact with dietary products in the intestine, it is of interest to investigate whether thylakoids might affect the composition of microbiota that confers benefits upon host well-being and health in a prebiotic way of action. In the present study thylakoids from spinach leaves were given to rats for $10 \mathrm{~d}$ and the intestinal microbiota were investigated, mainly with respect to bacteria such as lactobacilli and bifidobacteria, as well as harmful bacteria such as Enterobacteriaceae and Bacteroides. The influence of thylakoids on food intake was recorded, and the effect on metabolism was investigated through an oral glucose tolerance test (OGT'T). The long-term effects of thylakoid intake on insulin and glucose levels were also investigated.

\section{Experimental methods}

\section{Animals and experimental procedures}

The study was performed on rats (Rattus norvegicus) of the Sprague-Dawley strain (Mol: SPRD Han; Taconic M \& B A/S), bred under specific pathogen-free conditions with a controlled environment $\left(20 \pm 1^{\circ} \mathrm{C}, 50 \pm 10 \%\right.$ relative humidity, $12 \mathrm{~h}$ light-12 h dark cycle) and using an open cage system. After $7 \mathrm{~d}$ of acclimatisation, sixteen rats were kept individually and fed either a control diet (eight rats) or a thylakoid-enriched diet (eight rats) of $15 \mathrm{~g}$ to finish during the night for $10 \mathrm{~d}$. Besides the experimental diet, all rats were given $25 \mathrm{~g}$ of standard rat chow, for free eating during the day. The experimental diet was composed of standard rat chow ( $\mathrm{R} 36$; Lantmännen) enriched with either only rapeseed oil (control diet) or a thylakoid-oil suspension (thylakoid diet). Thylakoid membranes were extracted and purified from fresh baby-spinach leaves as described before ${ }^{(14,16)}$. The thylakoid diet was prepared by mixing $4 \mathrm{~g}$ thylakoid powder (corresponding to $132 \mathrm{mg}$ chlorophyll), $5 \mathrm{~g}$ rapeseed oil and $10 \mathrm{~g}$ water by using an Ultraturax mixer. The amount of thylakoids supplemented was calculated by the addition of $6 \mathrm{mg}$ chlorophyll per $\mathrm{g}$ of normal food intake $(22 \mathrm{~g}$ food/rat per d). Both thylakoid and control diets were isoenergetic, and had the energy (E) distribution of $25 \mathrm{E} \%$ carbohydrates, $60 \mathrm{E} \%$ fat and $15 \mathrm{E} \%$ proteins.

Normal rat chow was administered between 08.00 and 09.00 hours for free eating during the day. In the afternoon, between
16.00 and 18.00 hours, the remaining chow was removed and the consumption was recorded. The experimental diet of $15 \mathrm{~g}$ per rat was then administered, and in the next morning, any leftovers of the experimental diet were recorded. Body weight was measured every day during the $10-\mathrm{d}$ experimental time. On the first and last days, faecal samples were collected under clean conditions in the morning and the consistency of the faecal samples appeared to be similar for both groups. An OGTT was performed on the last day; a $15 \%$ glucose solution in a volume of $10 \%$ of the body weight was given as a bolus dose by gastric tube feeding. Blood for analysing the glucose concentration was collected by puncturing the tail vein at time points $0,15,30,45,60,90$ and $120 \mathrm{~min}$. The rat was then anaesthetised with isoflouran (Schering-Plogh a/s) and a laparotomy was performed. Tissue samples from the ileum, caecum and colon were collected, rinsed from loose materials with a cotton-compress and immediately placed in sterile tubes containing $3 \mathrm{ml}$ freezing media and frozen at $-80^{\circ} \mathrm{C}$. Blood for analysing the insulin concentration was obtained by a cardiac puncture, before the rat was killed, into tubes prepared with EDTA and Aprotinin (Trasylol; Bayer AG), centrifuged at $3000 \mathrm{~g}$ (Multifuge 1 Sorvall and Heraeus, Kendro Laboratory Products International Sales) for $15 \mathrm{~min}$ at $4^{\circ} \mathrm{C}$ before the plasma was collected and immediately frozen at $-80^{\circ} \mathrm{C}$. The study was approved by the Lund University Ethical Review Committee for Animal Experiments and was conducted according to the European Community's regulations concerning the protection of experimental animals.

\section{Analysis of blood glucose and plasma insulin}

Glucose levels were directly determined in the vein blood by using a Bayer's BREEZE ${ }^{\circledR}$ blood glucose meter (Bayer, Diabetes Care). Insulin was analysed with a sandwich immunoassay technique using double monoclonal antibodies directed at different sites on an insulin molecule (Mercodia).

\section{Analysis of faecal fat}

The faecal samples were kept in closed glass tubes and left to dry at room temperature in a fume cupboard for 1 month. The dry material was then weighed, placed in a mortar and homogenised together with chloroform (AnalaR Normapur ${ }^{\circledR}$; VWR International $\mathrm{AB}$ ) for $5 \mathrm{~min}$. The homogenised solution was transferred into a pre-weighed glass filter, and $100 \mathrm{ml}$ of chloroform was slowly washed through the glass filter for $10 \mathrm{~min}$. The glass filter, now containing the purified faecal components without fat, was dried overnight. The remaining material was weighed and the lost mass corresponds to the amount of fat that was present in the original faecal sample. The difference of fat content between day 0 and 10 was compared.

\section{Analysis of bacterial counts}

Conventional dilution procedures were used for the viable count of lactobacilli, bifidobacteria and Enterobactericeae in the mucosal samples from the ileum, caecum and colon, and in the faecal samples. Samples from appropriate dilutions were 
plated on Rogosa agar, modified Wilkins-Chalgren (MW) $\operatorname{agar}^{(17,18)}$ and violet red bile glucose agar (Oxoid). The Rogosa and MW agars were incubated at $37^{\circ} \mathrm{C}$ for $72 \mathrm{~h}$ anaerobically and the glucose agar was incubated at $37^{\circ} \mathrm{C}$ for $24 \mathrm{~h}$ aerobically, before the colony-forming units per $\mathrm{g}$ of tissue or faeces were calculated.

\section{5 rDNA sequencing}

To confirm the results on the viable count of lactobacilli and bifidobacteria, 16S rDNA sequencing was performed from isolates picked from MW agar and Rogosa plates. In total, 169 isolates (four to six from each rat) from colon and ileum samples were randomly picked (fifty-two colon isolates from MW agar plates, fifty-three colon isolates and sixty-four ileum isolates from Rogosa plates). As a template for the PCR, crude cell extracts were used as described by Quednau et $a l^{(19)}$. 16S rRNA genes were amplified by using the forward primer ENV1 and the reverse primer ENV2 (Table 1; Applied Biosystems). The PCR reaction mixture of total volume $25 \mu \mathrm{l}$ contained $0.4 \mu \mathrm{M}-\mathrm{ENV} 1,0.2 \mu \mathrm{M}-\mathrm{ENV} 2,2.5 \mu \mathrm{l} 10 \times$ PCR reaction buffer (500 mm-Tris- $\mathrm{HCl}, 100 \mathrm{~mm}-\mathrm{KCl}, 50$ mM- $\left.\left(\mathrm{NH}_{4}\right)_{2} \mathrm{SO}_{4}, 20 \mathrm{~mm}-\mathrm{MgCl}_{2}, \mathrm{pH} \quad 8 \cdot 3\right), 0 \cdot 2 \mu \mathrm{m}$-deoxyribonucleotide triphosphate, 2.5 units of FastStart Taq DNA polymerase (Roche Diagnostics) and $2 \mu$ of template DNA. PCR was performed in an Eppendorf Mastercycler ${ }^{\circledR} 5333$ using the following programme: $95^{\circ} \mathrm{C}$ for $3 \mathrm{~min}, 94^{\circ} \mathrm{C}$ for $3 \mathrm{~min}$, thirty cycles of $94^{\circ} \mathrm{C}$ for $1 \mathrm{~min}, 50^{\circ} \mathrm{C}$ for $45 \mathrm{~s}$ and $72^{\circ} \mathrm{C}$ for $2 \mathrm{~min}$, followed by an additional extension of $72^{\circ} \mathrm{C}$ for $7 \mathrm{~min}$. PCRs containing only reagents without sample DNA were run in parallel as negative PCR controls. PCR products $(2 \mu \mathrm{l})$ were verified on $1.5 \%$ agarose gel, and the amplification products were placed in ninety-six-well plates for sequencing at MWG-Biotech. The generated sequences, with a read length of 500-526 bases, were compared with the GenBank database (National Center for Biotechnology Information). The sequence similarity was between 98 and $100 \%$.

\section{DNA extraction of faeces and intestinal biopsies}

Faecal and intestinal segments of ileum and colon were thawed and placed in an ultrasonic bath (Millipore) for $5 \mathrm{~min}$, vortexed for $2 \mathrm{~min}$ and centrifuged at $12851 \mathrm{~g}$ (Eppendorf 5804R) for $10 \mathrm{~min}$. The supernatant was discarded and $190 \mu \mathrm{l}$ buffer
G2 and $15 \mu$ Proteinase $\mathrm{K}$ from the DNA Tissue Kit (Qiagen) were added to the intestinal samples. PBS (500 $\mu \mathrm{l} /$ $50 \mathrm{mg}$ ) was added to the faecal samples before all samples were incubated at $56^{\circ} \mathrm{C}$ overnight in a shaking water bath. After centrifugation at $12851 \mathrm{~g}$ (Eppendorf 5804R) for $8 \mathrm{~min}$, the solution was transferred to a Qiagen sample tube. Total DNA was extracted and eluted in $200 \mu \mathrm{l}$ buffer using Biorobot EZ1 (Qiagen) according to the manufacturer's instructions.

\section{Quantitative PCR analysis}

Bacterial groups were estimated using separate quantitative PCR (qPCR) assays. Each assay reaction contained $10 \mu \mathrm{l}$ QuantiTect ${ }^{\mathbb{B}}$ SYBR Green PCR Master Mix (Qiagen), $0 \cdot 5 \mu \mathrm{M}$ of each primer (Table 1), $2 \mu$ l of template DNA and RNase-free water to reach a final volume of $20 \mu$ l. Samples, standards and non-template controls were run as duplicates. Thermal cycling was carried out in Rotor-Gene Q (Qiagen) with a programme of $95^{\circ} \mathrm{C}$ for $15 \mathrm{~min}$, followed by forty cycles with denaturation at $95^{\circ} \mathrm{C}$ for $15 \mathrm{~s}$, annealing at $56-60^{\circ} \mathrm{C}$ for $30 \mathrm{~s}$ and elongation at $72^{\circ} \mathrm{C}$ for $30 \mathrm{~s}$. The fluorescent products were detected at the last step of each cycle. Melting curve analysis was done to ensure specific amplification. Absolute abundance of $16 \mathrm{~S}$ rRNA genes was calculated based on standard curves using Rotor-Gene Q series (Software 1.7; Qiagen). The detection limit was $10^{2}$ copies per reaction for all assays. To construct standard curves, cloned products from Lactobacillus plantarum DSM9843, Bifidobacterium infantis DSM15159, Escherichia coli CCUG29300 and Bacteroides prevotella were used. One loop of the cell suspension (cloned products) was transferred to $10 \mathrm{ml}$ of lysogeny broth medium with ampicillin and incubated overnight at $37^{\circ} \mathrm{C}$. DNA extraction was performed by using QIAprep ${ }^{\mathbb{B}}$ (Miniprep kit; Qiagen). The concentration of DNA $(\mathrm{ng} / \mu \mathrm{l})$ was finally measured with Nanodrop ND-1000 (Saveen Werner AB), and used for copy number calculation. Tenfold dilution series of the DNA products were made in TE buffer (10 mM-Tris; 1 mM-EDTA; pH 8.0). The numbers of bacteria were expressed as the numbers of amplicon copies per $\mathrm{g}$ wet weight of faeces or tissue.

\section{Terminal restriction fragment length polymorphism analysis}

$16 \mathrm{~S}$ rRNA genes were amplified as described above with the exception that the forward primer ENV1 was fluorescently

Table 1. Primers used in constructing standards of bacterial groups for quantitative PCR and for the amplification of $16 \mathrm{~S}$ rRNA genes

\begin{tabular}{|c|c|c|c|}
\hline Target group & Sequence $\left(5^{\prime}-3^{\prime}\right)$ & Amplicon size (bp) & Reference \\
\hline \multirow[t]{2}{*}{ Lactobacillus } & Lact-16S-F: GGAATCTTCCACAATGGACG & 216 & 20 \\
\hline & Lact-16S-R: CGCTTTACGCCCAATAAATCCGG & & \\
\hline \multirow[t]{2}{*}{ Bifidobacterium } & Bifido-F: TCGCGTCYGGTGTGAAAG & 243 & 21 \\
\hline & Bifido-R: CCACATCCAGCRTCCAC & & \\
\hline \multirow[t]{2}{*}{ Enterobacteriaceae } & Ec01457-F: CATTGACGTTACCCGCAGAAGAAGC & 195 & 22 \\
\hline & Eco1652-R: CTCTACGAGACTCAAGCTTGC & & \\
\hline \multirow[t]{2}{*}{ Bacteroides prevotella } & qBacPre-F: TTTATTGGGTTTAAAGGGAGCGTA & 166 & 23 \\
\hline & qBacPre-R: CAATCGGAGTTCTTCGTGATATCTA & & \\
\hline \multirow[t]{2}{*}{$16 S$ rRNA genes (ENV1, ENV2) } & E-coli 8-27-F: AGA GTT TGA TII TGG CTC AG & & 24 \\
\hline & E-coli 1511-1492-R: CGG ITA CCT TGT TAC GAC TT & & \\
\hline
\end{tabular}

F, forward; R, reverse. 
labelled with a fluorescent dye, FAM, at the $5^{\prime}$ end. Reactions were carried out in triplicate for each sample and a negative control was included in all PCR runs. PCR products were verified on $1.5 \%$ agarose gel. PCR products of each sample were pooled and further purified by using the MinElute PCR purification Kit (Qiagen). DNA was finally eluted in $15 \mu \mathrm{l}$ of washing buffer and DNA concentrations were measured with Nanodrop ND-1000 (Saveen Werner AB).

\section{Terminal restriction fragment length polymorphism analysis}

Aliquots (200 ng) of purified PCR products were digested at $37^{\circ} \mathrm{C}$ (Mastercycler ${ }^{\circledR} 5333$; Eppendorf) with either the restriction endonuclease enzyme $M s p \mathrm{I}$ for $5 \mathrm{~h}$ or $A l u \mathrm{I}$ for $2 \mathrm{~h}$ (Fermentas). Inactivation was made by heating at $65^{\circ} \mathrm{C}$ for $20 \mathrm{~min}$. After digestion, aliquots of the products were diluted four times with sterile water in a ninety-six-well plate. Samples were then sent to the DNA-lab (Skåne University Hospital, Malmö, Sweden) for terminal restriction fragment length polymorphism (T-RFLP) analysis in a capillary electrophoresis system. The data from the electrophoresis were analysed with GeneMapper ${ }^{\circledR}$ (version 4.0; Applied Biosystems) and the fragment sizes and peak areas were estimated using the Southern method (GeneMapper ${ }^{B}$ ). The size range was set from 30 to $600 \mathrm{bp}$. The peak amplitude threshold was set to fifty relative fluorescence units for samples and ten relative fluorescence units for the internal standard. The total peak area for each sample was calculated by summarising the area for all peaks in a sample. The individual relative peak area was expressed as percentage of the total area.

\section{Calculations}

The Shannon-Wiener index $\left(H^{\prime}\right)$ was calculated using relative peak area expressed as percentage of the total area for a sample by using the equation:

$$
H^{\prime}=-\sum p i \ln p i
$$

where $p i$ is the relative area expressed as percentage and $\ln$ is the natural logarithm ${ }^{(25)}$. Multivariate data analysis with principal component analysis (PCA) was performed in SIMCA-P+ (version 12.0.1; Umetrics) to reveal the possible differences in microbial population between the groups.

\section{Statistics}

Values are presented as means with their standard errors or as medians, ranges and 25th and 75th percentiles. The differences in glucose, insulin and counts of bacteria between the two experimental groups were assessed by a Mann-Whitney rank sum test using GraphPad Prism 4.0 (GraphPad Inc.). The difference in food intake, over the entire experimental period, was assessed by a two-way ANOVA analysis (GraphPad Prism 4.0). The incidences of different lactobacilli, bifidobacteria and other Gram-positive bacteria were evaluated by the Fisher exact test (Quick-Stat version 2.6). For all analyses, $P \leq 0.05$ was considered significant.

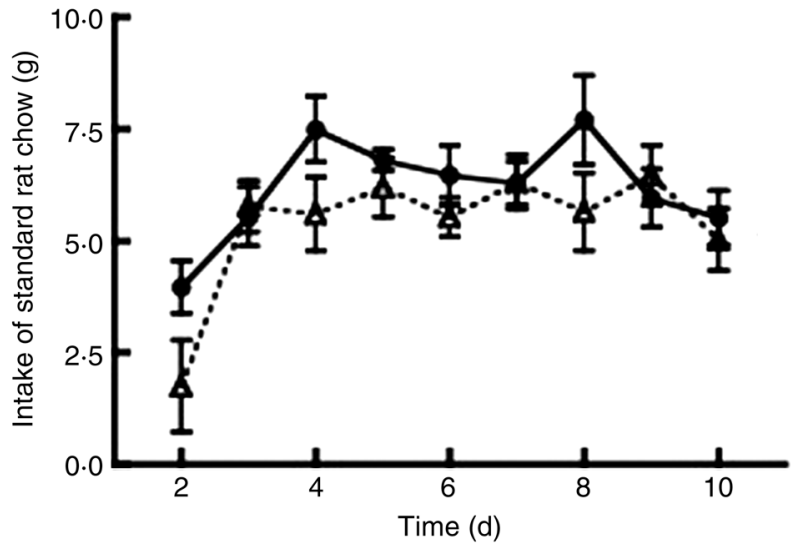

Fig. 1. Intake of standard rat chow during the $10 \mathrm{~d}$ experiment by animals fed a thylakoid-enriched diet $\left(--\boldsymbol{\Delta}_{-}-\right)$or a control diet $(-\bullet-)$. Values are means, with standard errors represented by vertical bars. Intake was significantly reduced in the thylakoid-fed animals compared with control animals $(P=0.003)$.

Results

Food intake, body weight, faecal fat, blood glucose and plasma insulin

All rats finished the experimental diets during the night. The intake of standard rat chow, i.e. the free eating of standard chow after finishing $15 \mathrm{~g}$ of experimental diet, was significantly decreased in the thylakoid-fed rats compared with the control-fed rats $(P=0.003$; Fig. 1$)$. No difference in body weight between the groups was observed. The content of faecal fat was unchanged in either the thylakoid or the control group from day 0 to day 10. Plasma insulin concentration at 120 min after the OGTT was significantly lower $(P=0.008)$ for the thylakoid group compared with the control group (Fig. 2). No significant difference was found for blood glucose concentrations after the OGT'T at any time point (Fig. 3).

\section{Bacterial quantification and identification}

Lactobacilli were significantly increased in the ileal mucosa in the thylakoid group compared with the control group, as demonstrated both by viable count $(P=0 \cdot 007$; Fig. 4$)$ and

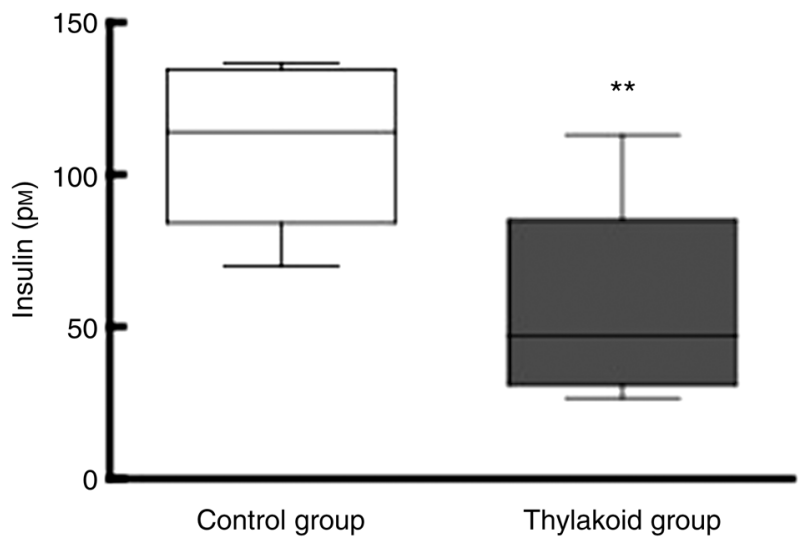

Fig. 2. Plasma concentration of insulin at $120 \mathrm{~min}$ after an oral glucose tolerance test in animals fed a thylakoid-enriched diet or a control diet for $10 \mathrm{~d}$. Values are medians, with ranges represented by vertical bars, and 25th and 75th percentiles represented by the box. ${ }^{\star *}$ Median value was significantly lower than that of the control group $(P=0.0082)$. 


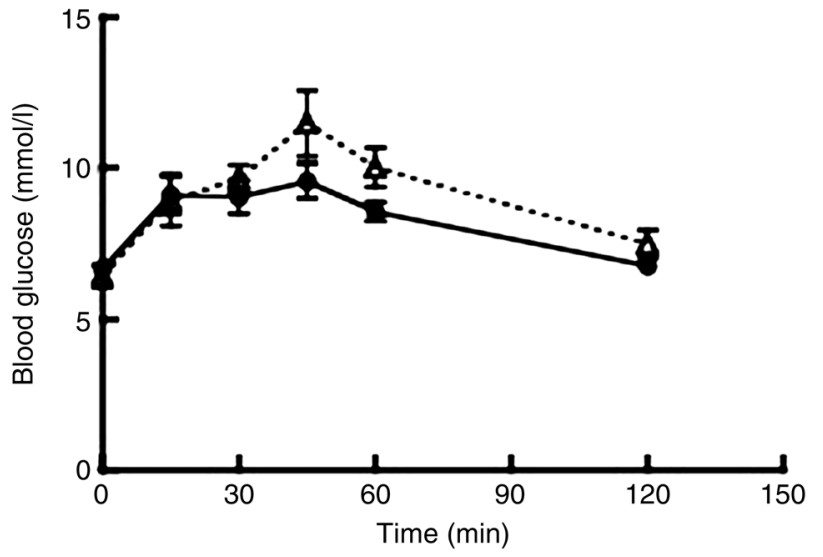

Fig. 3. Blood glucose levels at $0,15,30,45,60,90$ and 120 min after an oral glucose tolerance test in animals fed a thylakoid-enriched diet $(--\boldsymbol{\Delta}--)$ or a control diet (- - ) for $10 \mathrm{~d}$. Values are means, with standard errors represented by vertical bars. Mean values of the control and thylakoid groups were not significantly different at any time.

by qPCR $(P=0 \cdot 032$; Fig. 5). No difference was observed on the caecum or colonic mucosa. Moreover, a significant decrease of lactobacilli in the faecal samples of the thylakoid group was found with qPCR $(P=0 \cdot 007$; Fig. 5).

A significantly higher incidence of Lactobacillus reuteri $(P=$ 0.03 ) and a lower incidence of Lactobacillus johnsonii ( $P=$ $0 \cdot 003$ ) were found on the ileal mucosa for the thylakoid group compared with the control group by $16 \mathrm{~S}$ rDNA sequencing of isolates picked randomly from Rogosa agar plates (Table 2). In the colon mucosa, a significantly lower incidence of Lactobacillus jobnsonii $(P=0 \cdot 01)$ as well as a tendency for higher incidence of Lactobacillus reuteri $(P=0.09)$ were found for the thylakoid group compared with the control group (Table 2).

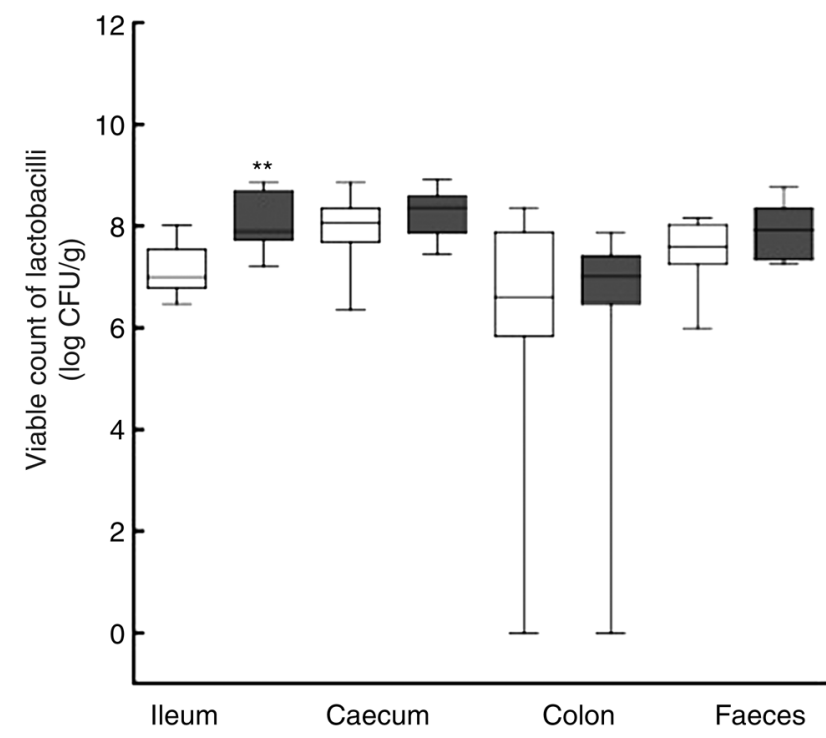

Fig. 4. Viable count (colony-forming units (CFU)/g tissue) of lactobacilli on the mucosa of the ileum, caecum and colon and in the faeces of animals fed a thylakoid-enriched diet ( $\bullet$ ) or a control diet $(\square)$ for $10 \mathrm{~d}$. Values are medians, with ranges represented by vertical bars, and 25th and 75th percentiles represented by the box. ${ }^{* *}$ Median value was significantly higher compared with that of the control group $(P=0.007)$. No differences were seen in the caecum, colon and faeces.

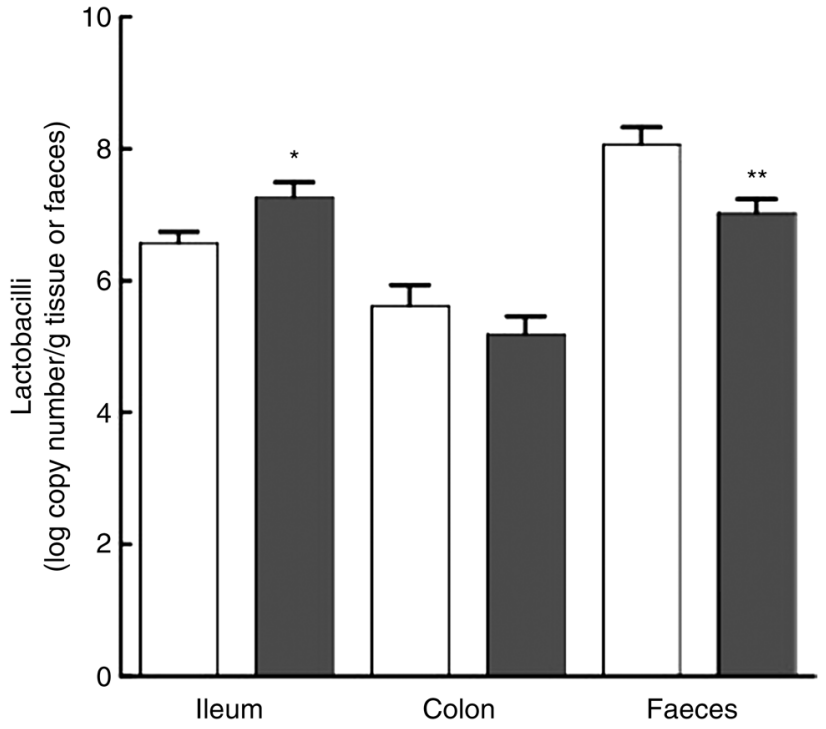

Fig. 5. Quantitative PCR analyses of lactobacilli on the ileum, colon mucosa and in the faeces (log number of amplicon copies/g wet weight) of animals fed a thylakoid-enriched diet ( $\bullet$ ) or a control diet $(\square)$ for $10 \mathrm{~d}$. Values are means, with standard errors represented by vertical bars. * Mean value was significantly higher than that of the control group $(P=0.032)$. ${ }^{* *}$ Mean value was significantly lower than that of the control group $(P=0.007)$. No difference was observed in the colon.

Analyses of bifidobacteria, on the mucosa of the ileum and colon and in the faeces by GPCR, showed similar results for both groups (data not shown). In the thylakoid group, a significantly decreased viable count on MW agar (claimed to be selective for bifidobacteria) was found in the faeces $(P=$ 0.0006), on the caecum $(P=0 \cdot 010)$ and on the colon mucosa $(P=0 \cdot 021)$, compared with the control group (Fig. 6). However, by $16 \mathrm{~S}$ rDNA sequencing of isolates picked randomly from MW agar plates of both groups, it was shown that the bifidobacteria represented less than $25 \%$ of these bacterial isolates. Due to insufficient selectivity of the traditional plating methods, the randomly picked bacteria from these plates were identified as Staphylococcus, Kocuria and Bacillus simplex (data not shown). Data from qPCR, sequencing and viable count indicate that bacteria that were decreased by thylakoids did not include bifidobacteria. Analyses of Enterobacteriaceae

Table 2. Incidence of different Lactobacillus spp. found by $16 \mathrm{~S}$ rDNA sequencing of isolates picked randomly from Rogosa agar plates of ileal and colonic mucosal samples

\begin{tabular}{|c|c|c|c|c|c|}
\hline & \multicolumn{2}{|c|}{ Control group } & \multicolumn{2}{|c|}{$\begin{array}{l}\text { Thylakoid } \\
\text { group }\end{array}$} & \multirow[b]{2}{*}{$P$} \\
\hline & Counts & $\%$ & Counts & $\%$ & \\
\hline \multicolumn{6}{|l|}{ Ileum } \\
\hline Lactobacillus reuteri & $11 / 29$ & 37.9 & $28 / 31$ & 90 & 0.03 \\
\hline Lactobacillus johnsonii & $17 / 29$ & 58.6 & $3 / 31$ & 10 & 0.003 \\
\hline Lactobacillus gasseri & $1 / 29$ & $3 \cdot 4$ & $0 / 31$ & 0 & NS \\
\hline Lactobacillus ruminis & $0 / 29$ & 0 & $0 / 31$ & 0 & NS \\
\hline \multicolumn{6}{|l|}{ Colon } \\
\hline Lactobacillus reuteri & $10 / 25$ & 40 & $21 / 25$ & 84 & 0.09 \\
\hline Lactobacillus johnsonii & $13 / 25$ & 52 & $2 / 25$ & 8 & 0.01 \\
\hline Lactobacillus gasseri & $0 / 25$ & 0 & $1 / 25$ & 4 & NS \\
\hline Lactobacillus ruminis & $2 / 25$ & 8 & $1 / 25$ & 4 & NS \\
\hline
\end{tabular}




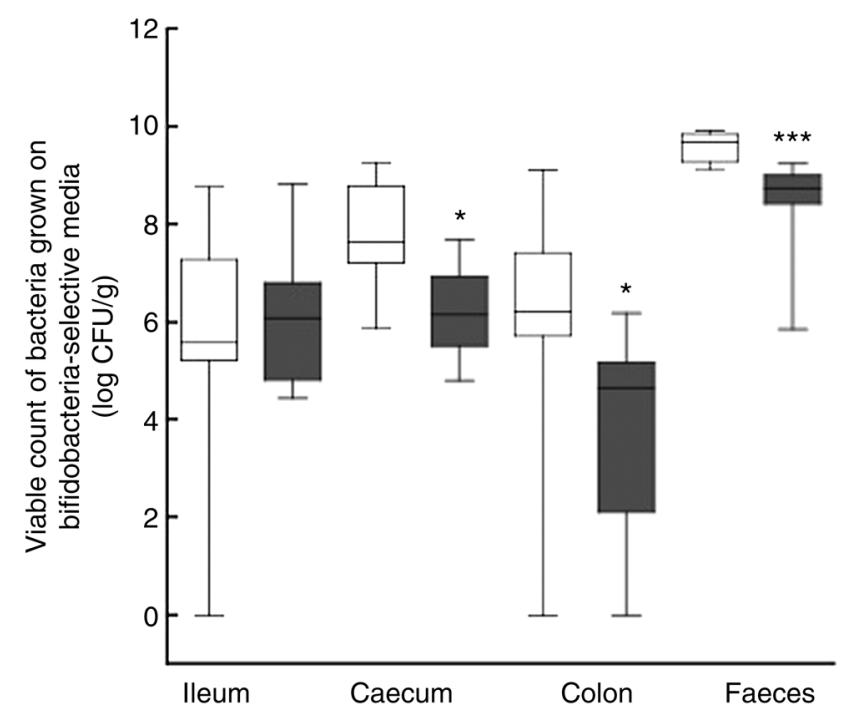

Fig. 6. Viable count (colony-forming units (CFU)/g tissue) of bacteria on modified Wikins-Chalgren (MW) media (claimed to be selective for bifidobacteria) on the mucosa of the ileum, caecum and colon and in the faeces of animals fed a thylakoid-enriched diet ( $(\mathbf{)})$ or a control diet ( $\square$ ) for $10 \mathrm{~d}$. Values are medians, with ranges represented by vertical bars, and 25th and 75th percentiles represented by the box. * Median value was significantly lower in the caecum $(P=0.0104)$ and colon $(P=0.0207)$ than that of the control group. *** Median value was significantly lower in the faeces $(P=0.0006)$ than that of the control group. No difference was observed in the ileum.

and Bacteroides resulted in no significant difference between the thylakoid and control groups.

Analyses of the microbiota with T-RFLP and PCA on the ileal and colon mucosa with both $M s p \mathrm{I}$ and $A l u \mathrm{I}$ for DNA digestion did not show any difference between the thylakoid and control groups (data not shown). In the faecal sample, however, different microbial compositions were observed in the two groups with PCA, as well as a more homogeneous microbiota in the thylakoid group compared with the control group (Fig. 7(a) and (b)). No significant differences between the thylakoid and control groups were found for bacterial diversity after calculations by the Shannon-Wiener diversity index (data not shown).

No significant differences between day 0 and day 10 were found in the faecal samples in either the thylakoid-fed or control-fed rats regarding Enterobacteriaceae and bifidobacteria.

\section{Discussion}

Addition of thylakoids to the diet for $10 \mathrm{~d}$ in the experimental rat model resulted in three main findings: the gut microbiota of the rat was modulated as lactobacilli were increased and supposedly harmful bacteria were reduced, the intake of food was decreased, and the insulin response after an OGTT was changed.

In the thylakoid group, an increased amount of ileal lactobacilli and a decreased amount of faecal lactobacilli were found, suggesting that thylakoids cause an increased ileal mucosal colonisation of lactobacilli. In fact, several studies have shown that specific strains of lactobacilli have the ability to colonise the intestinal mucosa in high numbers ${ }^{(26,27)}$. The identification of the lactobacilli by $16 \mathrm{~S}$ rDNA sequencing showed that Lactobacillus renteri was more pronounced in the ileal mucosa and, to a lesser extent, in colonic mucosa in the thylakoid group. Lactobacillus johnsonii was less pronounced in both ileal and colonic mucosa in the thylakoid group compared with the control group.

These novel findings demonstrate that small-intestinal mucosal microbiota can be affected by dietary supplementation, and specifically by the ingestion of thylakoid membranes. Recent studies have shown that Lactobacillus reuteri has health-promoting effects by restricting the growth of harmful bacteria ${ }^{(28-32)}$. Therefore, it is of interest that thylakoids were found to decrease Gram-positive bacteria such as Staphylococcus spp., Kocuria and Bacillus simplex.

The mechanism behind the beneficial effect of thylakoids in relation to microbiota is not known and needs to be further studied. There are two possible explanations. One is that the thylakoids themselves influence the growth of the bacteria in the intestine directly at the molecular level. The other explanation is an indirect effect of thylakoids, whereby a reduction in appetite and food intake may affect bacterial composition in the intestine.

Lactobacilli have been regarded as anti-obesity factors ${ }^{(4,5,10,11)}$. Elimination of Gram-positive bacteria from the gut appears to be important for achieving energy balance, e.g. Overweight children have an increased growth of Gram-positive bacteria such as Stapholycoccus aureus compared with normal-weight subjects ${ }^{(33,34)}$. Moreover, the lactobacilli species Lactobacillus rhamnosus GG and Lactobacillus gasseri have been found to reduce adiposity and inflammation associated with obesity ${ }^{(4,5)}$.

The anti-obesity effect of thylakoids can now be described both as an effect on appetite and energy metabolism and from now onwards, in addition, as a potential modulator of the gut microbiota.

The second main finding of this study was that thylakoid supplementation to the diet for $10 \mathrm{~d}$ decreased the intake of standard rat chow. This supports earlier findings of a longterm study with mice given free access to thylakoid-incorporated mouse chow, which resulted in decreased food intake, body weight and percentage of body fat, compared with control-fed mice ${ }^{(13)}$. The present decreased intake of standard rat chow in the thylakoid group indicates an increased satiety after supplementation of thylakoids. It can be speculated that these results are due to the deviated microbiota, since several studies in both animals and human subjects have shown interactions between the microbiota and body weight ${ }^{(35-37)}$. Prebiotics, i.e. non-bacterial components that beneficially affect the host by stimulating growth and/or activity of the microbiota, have been found to affect food intake and several gut hormones regulating satiety and hunger ${ }^{(38-40)}$. Since the supplementation of thylakoids has been found to give similar effects $^{(12-15,41)}$, they could be viewed as a prebiotic agent.

The third finding was a decreased insulin response after an OGTT at day 10 in the thylakoid group, while no difference in blood glucose levels was observed between the groups. Similar findings were reported in a short-term single-meal study in human subjects, where a reduced insulin response was found, although blood glucose levels were unchanged ${ }^{(12)}$. The 
(a)

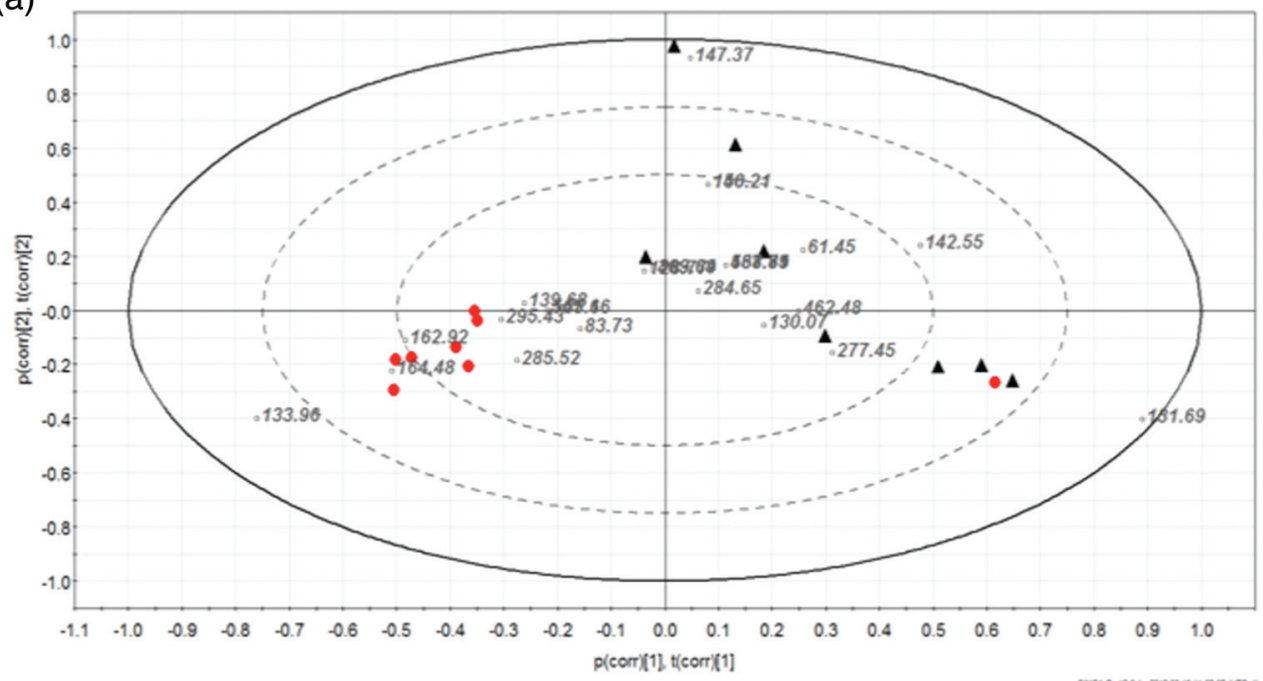

(b)

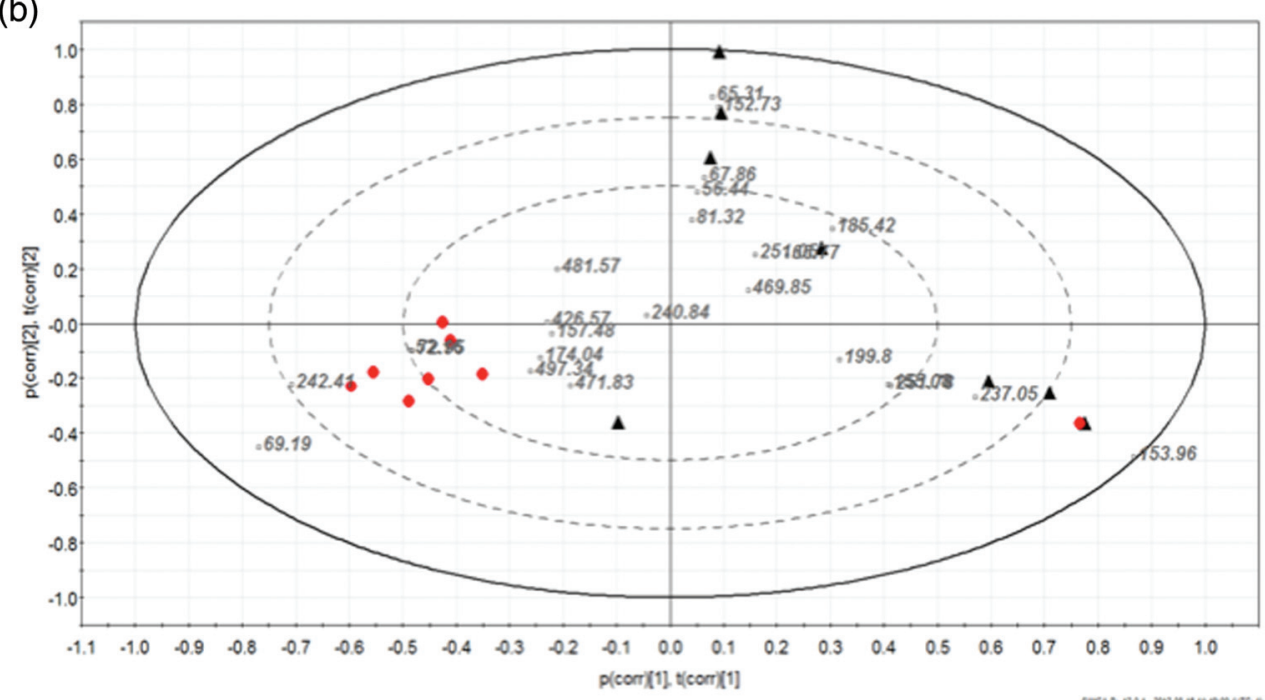

Fig. 7. Principal component analysis (PCA) of terminal restriction fragment length polymorphism (T-RFLP) data from (a) Mspl and (b) Alul digestion of bacterial DNA of faecal samples in the control animal $(\boldsymbol{\Delta})$ and thylakoid animal $(\bullet)$ groups. The microbiota in the faecal samples from the control and thylakoid animal groups are completely different from each other. (口), T-RFLP peaks (bacterial groups).

long-term effects of thylakoids decreasing insulin levels, presented here, have, however, never been shown before. A decreased passage of glucose during the experimental period of $10 \mathrm{~d}$ would theoretically result in a lower secretion of insulin, resulting in higher insulin sensitivity. It has previously been reported that thylakoids, in a dose-dependent way, decrease the in vitro uptake of methyl-glucose over the rat intestinal wall ${ }^{(16)}$. The reason for this decrease could be either an indirect cause of a steric hindrance formed by thylakoids binding to the mucosa of the intestine, or less likely, a direct cause of thylakoids binding to glucose molecules ${ }^{(16)}$. Also, the barrier of the intestine has been proposed to be important for preventing diabetes, where an increased permeability of the intestine might lead to insulin resistance ${ }^{(42)}$. The formation of an intestinal steric hindrance by thylakoids could thereby reinforce the intestinal wall. However, since the glucose uptake was the same in thylakoid-fed and control-fed animals, this explanation seems less likely.
Another explanation for the decreased insulin response could be the observed modulation of the microbiota. Recent studies have proposed that the microbiota modulate lipid and glucose homeostasis, by affecting the metabolism in the liver and adipose tissue $^{(43)}$. Furthermore, the compositions of the microbiota have been found to affect the down- or up-regulation of gene expression, which may promote adiposity ${ }^{(9)}$ or decrease insulin and blood glucose concentrations ${ }^{(44)}$. Moreover, several strains of lactobacilli and bifidobacteria have been reported to have antidiabetic effects, by preventing elevation of blood glucose and reducing insulin responses ${ }^{(44-47)}$. The explanation for these effects, however, is mainly prevention of immune-mediated destruction of pancreatic $\beta$-cells. Thylakoids have, in a previous mouse experiment, been found to reduce insulin levels and adiposity ${ }^{(13)}$. This could hypothetically be a result of a changed microbiota, through interaction with the adipose tissue.

To conclude, the addition of thylakoids to the diet for $10 \mathrm{~d}$ affects the intestinal composition of the microbiota by 
increasing the ileal colonisation of lactobacilli. At the same time thylakoids were found to reduce food intake as well as insulin levels after an OGTT, without affecting glucose levels. The reduced insulin levels may be a consequence of the changed intestinal composition of the microbiota. More studies of the interaction of thylakoids with the microbiota, and the question whether thylakoids can be regarded as a prebiotic agent, remain to be investigated further.

\section{Acknowledgements}

The authors are grateful for the financial support from FORMAS, VINNOVA, the Carl Trygger Foundation, the Royal Physiographic Society of Lund, and the Swedish Medical Research Council, for the donation of spinach from SABA Fresh, and for the assistance rendered by Jie Xie in performing microbial analysis and by Linda Ahlkvist in performing the insulin analysis. Both C. M. and N. O. were equally responsible for planning the study, performing animal experiments and writing the paper. N. O. performed the microbial analysis. B. W. assisted in planning the study, in performing the experiments and in writing the paper. S. A. and G. M. assisted in performing the analyses. P.-A. A. assisted in writing the paper. C. E.-A. assisted in planning the study and in writing the paper.

The authors have no conflict of interest.

\section{References}

1. Hill J, Wyatt H, Reed G, et al. (2003) Obesity and the environment: where do we go from here? Science 299, 853-855.

2. Ginsberg HN (2000) Insulin resistance and cardiovascular disease. J Clin Invest 106, 453-458.

3. van Hees AMJ, Saris WHM, Dallinga-Thie GM, et al. (2008) Fasting and postprandial remnant-like particle cholesterol concentrations in obese participants are associated with plasma triglycerides, insulin resistance, and body fat distribution. J Nutr 138, 2399-2405.

4. Kadooka Y, Sato M, Imaizumi K, et al. (2010) Regulation of abdominal adiposity by probiotics (Lactobacillus gasseri SBT2055) in adults with obese tendencies in a randomized controlled trial. Eur J Clin Nutr 64, 636-643.

5. Andrade S \& Borges N (2009) Effect of fermented milk containing Lactobacillus acidophilus and Bifidobacterium longum on plasma lipids of women with normal or moderately elevated cholesterol. J Dairy Res 76, 469-474.

6. Furtado M (2009) Probiotics, prebiotics, gut microbiota, and obesity. Bariatic Times 11, 27-30.

7. Bajzer M \& Seeley RJ (2006) Physiology: obesity and gut flora. Nature 444, 1009-1010.

8. Ley RE, Bäckhed F, Turnbaugh P, et al. (2005) Obesity alters gut microbial ecology. Proc Natl Acad Sci U S A 102, 11070-11075.

9. Bäckhed F, Ding H, Wang T, et al. (2004) The gut microbiota as an environmental factor that regulates fat storage. Proc Natl Acad Sci U $S$ A 101, 15718-15723.

10. Taranto MP, Medici M, Perdigon G, et al. (2000) Effect of Lactobacillus reuteri on the prevention of hypercholesterolemia in mice. J Dairy Sci 83, 401-403.

11. Luoto R, Kalliomäki M, Laitinen K, et al. (2010) The impact of perinatal probiotic intervention on the development of overweight and obesity: follow-up study from birth to 10 years. Int J Obes (Lond) 34, 1531-1537.

12. Köhnke R, Lindbo A, Larsson T, et al. (2009) Thylakoids promote release of the satiety hormone cholecystokinin while reducing insulin in healthy humans. Scand J Gastroenterol 44, 712-719.
13. Köhnke R, Lindqvist A, Göransson N, et al. (2009) Thylakoids suppress appetite by increasing cholecystokinin resulting in lower food intake and body weight in high-fat fed mice. Phytother Res 23, 1778-1783.

14. Emek SC, Szilagyi A, Akerlund H-E, et al. (2010) A large scale method for preparation of plant thylakoids for use in body weight regulation. Prep Biochem Biotechnol 40, 13-27.

15. Albertsson P- $\AA$, Köhnke R, Emek SC, et al. (2007) Chloroplast membranes retard fat digestion and induce satiety: effect of biological membranes on pancreatic lipase/co-lipase. Biochem J 401, $727-733$.

16. Montelius C, Gustafsson K, Weström B, et al. (2011) Chloroplast thylakoids reduce glucose uptake and decrease intestinal macromolecular permeability. Br J Nutr 106, 836-844.

17. Osman N, Adawi D, Molin G, et al. (2006) Bifidobacterium infantis strains with and without a combination of oligofructose and inulin $(\mathrm{OFI})$ attenuate inflammation in DSS-induced colitis in rats. BMC Gastroenterol 6, 31.

18. Rada V \& Petr J (2000) A new selective medium for the isolation of glucose non-fermenting bifidobacteria from hen caeca. J Microbiol Methods 43, 127-132.

19. Quednau M, Ahrné S, Petersson AC, et al. (1998) Identification of clinically important species of Enterococcus within 1 day with randomly amplified polymorphic DNA (RAPD). Curr Microbiol 36, 332-336.

20. Nordin N, Abdulamir A \& Bakar F (2010) Methods for precise molecular detection of probiotic microflora: using adjusted molecular biology protocols, primer sets and PCR assays. J Biotechnol 9, 25-32.

21. Rinttilä TT, Kassinen AA, Malinen EE, et al. (2003) Development of an extensive set of $16 \mathrm{~S}$ rDNA-targeted primers for quantification of pathogenic and indigenous bacteria in faecal samples by real-time PCR. J Appl Microbiol 97, 1166-1177.

22. Bartosch S, Fite A, Macfarlane GT, et al. (2004) Characterization of bacterial communities in feces from healthy elderly volunteers and hospitalized elderly patients by using real-time PCR and effects of antibiotic treatment on the fecal microbiota. Appl Environ Microbiol 70, 3575-3581.

23. Okabe S, Okayama N, Savichtcheva O, et al. (2007) Quantification of host-specific Bacteroides-Prevotella 16S rRNA genetic markers for assessment of fecal pollution in freshwater. Appl Microbiol Biotechnol 74, 890-901.

24. Brosius J, Palmer ML, Kennedy PJ, et al. (1978) Complete nucleotide sequence of a $16 \mathrm{~S}$ ribosomal RNA gene from Eschericbia coli. Proc Natl Acad Sci U S A 75, 4801-4805.

25. Magurran AE (2004) Measuring biological diversity. Afr J Aquat Sci 29, 285-286.

26. Servin AL \& Coconnier M-H (2003) Adhesion of probiotic strains to the intestinal mucosa and interaction with pathogens. Best Pract Res Clin Gastroenterol 17, 741-754.

27. Johansson ML, Molin G, Jeppsson B, et al. (1993) Administration of different Lactobacillus strains in fermented oatmeal soup: in vivo colonization of human intestinal mucosa and effect on the indigenous flora. Appl Environ Microbiol 59, 15-20.

28. Casas IA \& Dobrogosz WJ (2000) Validation of the probiotic concept: Lactobacillus reuteri confers broad-spectrum protection against disease in humans and animals. Microb Ecol Health Dis 12, 247-285.

29. Cleusix V, Lacroix C, Vollenweider S, et al. (2008) Glycerol induces reuterin production and decreases Escherichia coli population in an in vitro model of colonic fermentation with immobilized human feces. FEMS Microbiol Ecol 63, 56-64.

30. Turner MS, Lo R \& Giffard PM (2007) Inhibition of Staphylococcus aureus growth on tellurite-containing media by Lactobacillus reuteri is dependent on CyuC and thiol production. Appl Environ Microbiol 73, 1005-1009

31. Gan BS, Kim J, Reid G, et al. (2002) Lactobacillus fermentum RC-14 inhibits Staphylococcus aureus infection of surgical implants in rats. J Infect Dis 185, 1369-1372. 
32. Laughton JMJ, Devillard EE, Heinrichs DED, et al. (2006) Inhibition of expression of a staphylococcal superantigen-like protein by a soluble factor from Lactobacillus renteri. Microbiology 152, 1155-1167.

33. Kalliomäki M, Collado MC, Salminen S, et al. (2008) Early differences in fecal microbiota composition in children may predict overweight. Am J Clin Nutr 87, 534-538.

34. DiBaise JK, Zhang H, Crowell MD, et al. (2008) Gut microbiota and its possible relationship with obesity. Mayo Clin Proc 83, 460-469.

35. Chang BJ, Park SU, Jang YS, et al. (2011) Effect of functional yogurt NY-YP901 in improving the trait of metabolic syndrome. Eur J Clin Nutr 65, 1250-1255.

36. Mallappa RH, Rokana N, Duary RK, et al. (2012) Management of metabolic syndrome through probiotic and prebiotic interventions. Indian J Endocrinol Metab 16, 20-27.

37. An HM, Park SY, Lee DK, et al. (2011) Antiobesity and lipid-lowering effects of Bifidobacterium spp. in high fat diet-induced obese rats. Lipids Health Dis 10, 116.

38. Cani PD, Lecourt E, Dewulf EM, et al. (2009) Gut microbiota fermentation of prebiotics increases satietogenic and incretin gut peptide production with consequences for appetite sensation and glucose response after a meal. Am. J Clin Nutr 90, 1236-1243.

39. Cani PD, Neyrinck AM, Maton N, et al. (2005) Oligofructose promotes satiety in rats fed a high-fat diet: involvement of glucagonlike peptide-1. Obes Res 13, 1000-1007.
40. Reimer RA \& Russell JC (2008) Glucose tolerance, lipids, and GLP-1 secretion in JCR:LA-cp rats fed a high protein fiber diet. Obesity 16, 40-46.

41. Köhnke R, Svensson L, Piedra JLV, et al. (2010) Feeding appetite suppressing thylakoids to pigs alters pancreatic lipase/colipase secretion. Livest Sci 134, 68-71.

42. de Kort S, Keszthelyi D \& Masclee AAM (2011) Leaky gut and diabetes mellitus: what is the link? Obes Rev 12, 449-458.

43. Delzenne NM \& Cani PD (2011) Interaction between obesity and the gut microbiota: relevance in nutrition. Annu Rev Nutr 31, $15-31$.

44. Kondo S, Xiao J-Z, Satoh T, et al. (2010) Antiobesity effects of Bifidobacterium breve strain B-3 supplementation in a mouse model with high-fat diet-induced obesity. Biosci Biotechnol Biochem 74, 1656-1661.

45. Matsuzaki TT, Yamazaki RR, Hashimoto SS, et al. (1997) Antidiabetic effects of an oral administration of Lactobacillus casei in a non-insulin-dependent diabetes mellitus (NIDDM) model using KK-Ay mice. Endocr J 44, 357-365.

46. Tabuchi M, Ozaki M, Tamura A, et al. (2003) Antidiabetic effect of Lactobacillus $G G$ in streptozotocin-induced diabetic rats. Biosci Biotechnol Biochem 67, 1421-1424.

47. Yadav H, Jain S \& Sinha PR (2008) Oral administration of dahi containing probiotic Lactobacillus acidopbilus and Lactobacillus casei delayed the progression of streptozotocin-induced diabetes in rats. J Dairy Res 75, 189-195. 